\title{
Spintronic properties of zigzag-edged triangular graphene flakes
}

H. Şahin, R. T. Senger, and S. Ciraci

Citation: Journal of Applied Physics 108, 074301 (2010);

View online: https://doi.org/10.1063/1.3489919

View Table of Contents: http://aip.scitation.org/toc/jap/108/7

Published by the American Institute of Physics

\section{Articles you may be interested in}

Magnetization of graphane by dehydrogenation

Applied Physics Letters 95, 222510 (2009); 10.1063/1.3268792

Making a field effect transistor on a single graphene nanoribbon by selective doping

Applied Physics Letters 91, 253122 (2007); 10.1063/1.2826547

Electronic transport in locally gated graphene nanoconstrictions Applied Physics Letters 91, 192107 (2007); 10.1063/1.2803074

The peculiar transport properties in $\mathrm{p}-\mathrm{n}$ junctions of doped graphene nanoribbons Journal of Applied Physics 110, 013718 (2011); 10.1063/1.3605489

Intrinsic magnetism of monolayer graphene oxide quantum dots Applied Physics Letters 108, 033105 (2016); 10.1063/1.4940054

Switching and rectification of a single light-sensitive diarylethene molecule sandwiched between graphene nanoribbons

The Journal of Chemical Physics 135, 184703 (2011); 10.1063/1.3657435

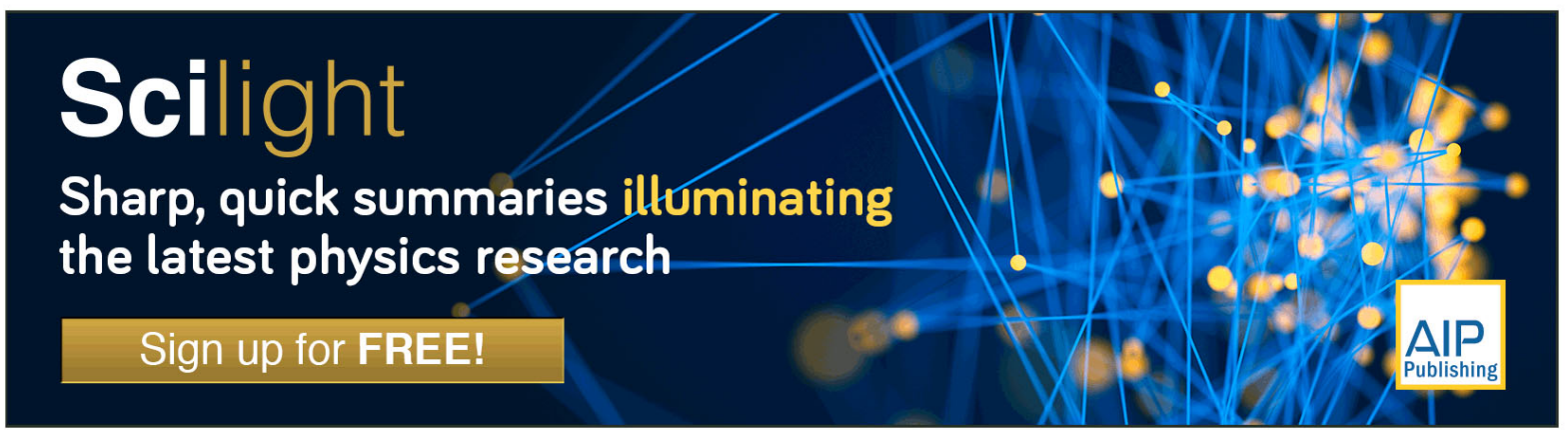




\title{
Spintronic properties of zigzag-edged triangular graphene flakes
}

\author{
H. Şahin, ${ }^{1, a)}$ R. T. Senger, ${ }^{2, b)}$ and S. Ciraci ${ }^{1,3, c)}$ \\ ${ }^{1}$ UNAM-Institute of Materials Science and Nanotechnology, Bilkent University, 06800 Ankara, Turkey \\ ${ }^{2}$ Department of Physics, Izmir Institute of Technology, 35430 Izmir, Turkey \\ ${ }^{3}$ Department of Physics, Bilkent University, 06800 Ankara, Turkey
}

(Received 31 May 2010; accepted 13 August 2010; published online 1 October 2010)

\begin{abstract}
We investigate quantum transport properties of triangular graphene flakes with zigzag edges by using first principles calculations. Triangular graphene flakes have large magnetic moments which vary with the number of hydrogen atoms terminating its edge atoms and scale with its size. Electronic transmission and current-voltage characteristics of these flakes, when contacted with metallic electrodes, reveal spin valve and remarkable rectification features. The transition from ferromagnetic to antiferromagnetic state under bias voltage can, however, terminate the spin polarizing effects for specific flakes. Geometry and size dependent transport properties of graphene flakes may be crucial for spintronic nanodevice applications. (C) 2010 American Institute of Physics.
\end{abstract} [doi:10.1063/1.3489919]

\section{INTRODUCTION}

Graphene and graphene based nanostructures are focus of intensive research activities due to their impressive material properties ${ }^{1-6}$ and promising application potential ${ }^{7-12}$ in novel electronic devices. In particular, edge-localized spin polarizations found in graphene ribbons, ${ }^{13,14}$ flakes, ${ }^{15-17}$ and at defect sites ${ }^{18,19}$ introduce magnetic properties that can be utilized for spintronic applications. Recent studies have also revealed the ferromagnetic ground state of graphene nanodots, ${ }^{20}$ triangular shaped graphene fragments ${ }^{21-24}$ and graphene domains on two-dimensional (2D) hydrocarbons, ${ }^{25}$ and the possibility of observing spin polarized currentvoltage characteristics of such graphene flakes.

While pristine graphene provides high carrier mobility and ambipolar behavior, semiconductor nanoscale materials having tunable band gap are more desirable from the perspective of potential nanoelectronics applications. In this context, recent efforts have been devoted to precise controlling electronic and magnetic properties of graphene sheets by functionalization via adatom adsorption. The synthesis of a 2D hydrocarbon in honeycomb structure, namely, graphane, ${ }^{26-29}$ is one of the successful example for such functionalization. Very recently, we reported the possibility of obtaining tunable band gap and magnetization through dehydrogenation of domains on 2D graphane and graphane nanoribbons. $^{25,30}$ Stability and electronic properties of graphene flakes uniformly functionalized by methyl $\left(\mathrm{CH}_{3}\right)$, phenyl $\left(\mathrm{C}_{6} \mathrm{H}_{5}\right)$, and nitrophenyl $\left(\mathrm{C}_{6} \mathrm{H}_{4} \mathrm{NO}_{2}\right)$ groups were also discussed earlier. ${ }^{31-33}$

Recent experimental observations ${ }^{34-38}$ and theoretical studies $^{39-41}$ show that the electronic, magnetic, and conductance properties of graphitic fragments can be changed significantly upon the termination of their edges. In addition to purely zigzag and armchair edged graphene, experimental

\footnotetext{
${ }^{a)}$ Electronic mail: shasan@bilkent.edu.tr.

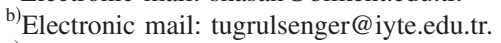

${ }^{\mathrm{c})}$ Electronic mail: ciraci@fen.bilkent.edu.tr.
}

verification of the existence of alternating series of zigzag and armchair segments at the edges and energetics of reconstructions have also been reported. ${ }^{42}$ Originating from the antiferromagnetic ground state of zigzag edges, adatom, ${ }^{15}$ and topology ${ }^{43}$ dependent trends in electronic properties of rectangular flakes have also investigated.

In this work, we study graphene flakes having equilateral triangular shapes with zigzag edges ( $n$-TGF), where $n$ denotes the number of edge hexagonal cells in one side of the triangle. The flakes have been considered as bare $\left(\mathrm{C}_{n^{2}+4 n+1}\right)$, each edge atom being saturated with one $\left(\mathrm{C}_{n^{2}+4 n+1} \mathrm{H}_{3 n+3}\right)$ or two hydrogen atoms $\left(\mathrm{C}_{n^{2}+4 n+1} \mathrm{H}_{6 n+6}\right)$. We find that these flakes have large spin magnetic moment values of $4(n-1)$, $(n-1)$, and $2(n-1)$, respectively, in units of $\mu_{B}$. When these triangular graphene flakes (TGFs) have been contacted with thin metallic electrodes we calculate that the current running through them gets both spin polarized and rectified.

\section{CALCULATION METHODS}

Optimization of geometrical structures of TGFs and calculations of their magnetic and electric properties are performed by using the software package ATOMISTIX TOOLKIT (ATK) (Ref. 44) based on density functional theory (DFT). The spin-dependent exchange-correlation potential is approximated within the generalized gradient approximation. The criteria of convergence used for total energy and Hellman-Feynman forces were $10^{-4} \mathrm{eV}$ and $0.005 \mathrm{eV} / \AA$, respectively. The electrostatic potentials were determined on a real-space grid with a mesh cutoff energy of 150 Ry. Double-zeta-polarized basis sets of local numerical orbitals were employed to increase the accuracy of our calculations.

For determination of quantum transport properties of the electrode-TGF-electrode system, ATK use nonequilibrium Green's function formalism. Transport calculations are performed with the Brillouin zone sampled with $(1,1,51)$ points within the Monkhorst-Pack $k$-point sampling scheme. The current through the TGFs is determined by summing the transmission probabilities for electron states from one elec- 
trode to another within the energy window $\mu_{L}-\mu_{R}=|e V|$, where $\mu_{L}\left(\mu_{R}\right)$ is the electrochemical potential of the left (right) electrode under the applied bias $V$. Therefore, the spin-dependent current is given by the formula

$$
I_{\sigma}(V)=G_{0} \int_{\mu_{L}}^{\mu_{R}} T_{\sigma}(E, V) d E,
$$

where $G_{0}=e^{2} / h$ is the quantum conductance unit and $T_{\sigma}(E, V)$ is the quantum mechanical transmission probability for electrons with spin state $\sigma$ and energy $E$. During the self-consistent calculation of $I-V$ spectrum charge on the TGFs is not fixed and energy is minimized with respect to the electrochemical potentials of the electrodes at each voltage increment. In order to achieve convergence of the electronic states with increasing voltages to the desired level of accuracy, calculations performed within the bias window (from -1 to $+1 \mathrm{~V}$ ) in steps of $0.005 \mathrm{~V}$. By using the carbon chains attached to the TGF, it is ensured that the screening occurs in the device region.

\section{RESULTS}

\section{A. Atomic and magnetic ground state properties of TGFs}

First, we have performed geometry optimizations of TGFs and determined the spin polarized charge density of the optimized structures. Due to the particular shape of the TGFs spin-relaxed calculation leads to lower ground state energies compared to those of spin-unrelaxed calculation; here we have found that ferromagnetically ordered spin accumulation at the edges gives a nonzero magnetic moment to the flake. Apart from minor bond contractions of the edge atoms TGFs preserve their regular hexagonal structure even when the flakes are not hydrogenated. Saturating the edge carbon atoms with either one or two hydrogen atoms considerably modifies the magnetic ground state, by altering the total magnetic moment.

Graphene, with its hexagonal lattice structure resulting from $s p^{2}$-type hybridization of carbon atoms, is a planar $\pi$ conjugated system. It can be viewed as made up of $A$ and $B$ sublattices of carbon atoms. Repulsive case of the Lieb's theorem ${ }^{45}$ reveals uniqueness of the ground state and provides a simple formula for calculating the magnetic ground state of such bipartite systems. According to the rule provided by the theorem, total net spin magnetization of a graphene structure is given by

$$
\mu=\frac{1}{2}\left|N_{A}-N_{B}\right| g \mu_{B},
$$

where $g \approx 2$ for electron, $\mu_{B}$ is Bohr magneton, and $N_{A}$ and $N_{B}$ denote the number of carbon atoms in $A$ and $B$ sublattices, respectively. Each carbon atom in graphene is connected to the nearest neighbors by three covalent bonds, while leaving behind a $p_{z}$-orbital electron contributing to the spin magnetic moment. Such electrons in each sublattice have opposite spin states and make spin paired $\pi$ bonds, thus total spin magnetic moment of the system is zero unless a difference is created in the numbers of atoms of the $A$ and $B$ sublattices. In some graphene flakes, such as those having equilateral triangular shapes and zigzag edges, $N_{A}$ and $N_{B}$ are

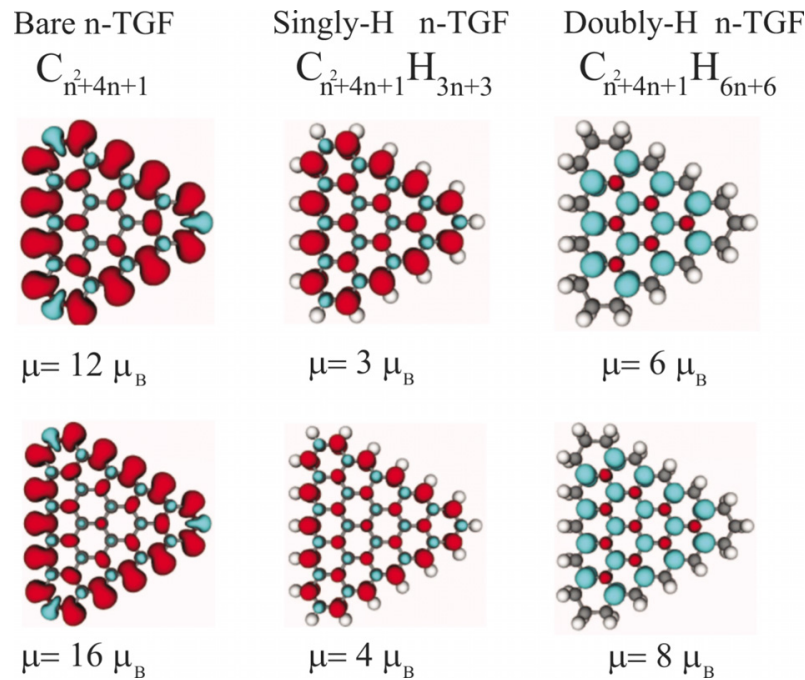

FIG. 1. (Color online) Atomic structure and isosurface of charge density difference of spin-up $(\uparrow)$ and spin-down $(\downarrow)$ states for 4-(upper row) and 5-triangle (lower row) graphene flakes: Bare, singly, and doubly hydrogenated edges. Calculated net magnetic moments of the flakes are given in terms of Bohr magneton $\left(\mu_{B}\right)$. Difference charge density of spin-up and spin-down states is shown by red (dark) and blue (light) isosurface, respectively.

different and leads to finite net spin magnetic moments. ${ }^{22,23}$ Moreover, in bare (not hydrogenated) flakes the edge carbon atoms that make only two covalent bonds contribute to the spin moment with two nonbonding electrons.

In Fig. 1, we present the optimized structures and spin charge density difference $\left(\Delta \rho=\rho_{\uparrow}-\rho_{\downarrow}\right)$ isosurface for bare, singly, and doubly hydrogenated cases of $n$-TGFs $(n=4,5)$. In general, we see that each carbon atom has an induced spin imbalance opposite to its nearest neighbors, however the spin polarization of hydrogen atoms is negligible.

Calculated total spin magnetic moments of the structures using DFT are in integer multiples of $\mu_{B}$ and either verifies the results of Lieb's theorem or can be understood by simple modifications of it. In the flakes with singly hydrogenated edges, all the carbon atoms are coordinated as reminiscent of infinite graphene, and $\mu / \mu_{B}=N_{A}-N_{B}=n-1$ in accordance with Lieb's theorem. When the hydrogen atoms are removed from the flake (bare flake case) the $3 n$ atoms of sublattice $A$ and three atoms of sublattice $B$ each have an extra nonbonding electron that contributes to the magnetic moment, consequently giving $\mu / \mu_{B}=n-1+3 n-3=4(n-1)$. On the other hand, in doubly hydrogenated flakes, all the edge carbon atoms have $s p^{3}$ hybridized electrons with vanishing contribution to spin magnetic moment. Thus, in this case $\mu / \mu_{B}$ $=|n-1-3 n+3|=2(n-1)$, where the direction of magnetic moment is reversed, and the net moment of the system increases with respect to the singly hydrogenated case. Tunability of the spin magnetic moments of TGFs through degree of hydrogenation is an interesting feature that may be utilized for nanodevice applications. Saturation of TGF edges by other atoms may give rise to similar modifications in their electronic and magnetic structures.

\section{B. Transport properties of TGFs}

Next, we consider electrical conductance and I-V characteristics of TGFs when contacted with metallic electrodes. 


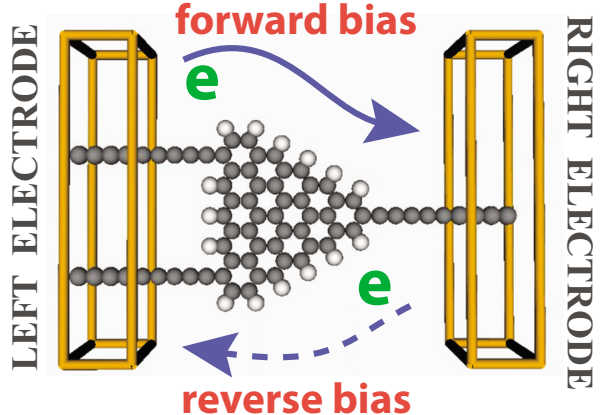

FIG. 2. (Color online) Electrode-device geometry and convention for forward and reverse bias applied to TGFs.

We use linear carbon chains as model electrodes. Carbon atomic chains which are known to be metallic ${ }^{15,46}$ are expected to make reasonably good contacts with the flakes. As an alternative to carbon-chain electrodes, earlier the robustness of our conductance calculations was also tested by using semi-infinite gold bar electrodes and consistent results were obtained for rectangular flakes. ${ }^{15}$ At the contact sites, the introduced hydrogen atoms are removed and connection between electrode and flake carbon atoms is established through double bonds. Due to asymmetric form of deviceelectrode geometry one expects different current profiles for forward and reverse bias voltages. The convention we have adopted is schematically shown in Fig. 2, where forward bias corresponds to flow of electrons from the left to the right electrode, i.e., the current is flowing from right to left electrode.
For the ballistic current of electrons, the spin-dependent transmission probability from one electrode to other strongly depends on the eigenstates of the TGF molecule. The molecular orbitals perfectly coupled to the electrodes behave as an open channel and provides propagation with minimal scattering through the TGF and hence the magnitude of the transmission coefficient is determined by electrode-device interaction. Eigenstates within the $E_{F} \pm 1 \mathrm{eV}$ energy window, indicating the highest-occupied-molecular-orbital (HOMO) and lowest-unoccupied-molecular-orbital (LUMO) of singly hydrogenated and bare $n$-TGFs $(n=4,5)$ are presented in Fig. 3(a). It is obvious that the HOMO-LUMO gap $(\Delta)$ gets narrower upon the hydrogenation of TGFs. Resulting from the ferromagnetic ground state, within the $E_{F} \pm 1 \mathrm{eV}$ energy window up and down spin states are wellseparated around the $E_{F}$. In the energy window used for Fig. 3(a), all the filled levels are for up-spin states, whereas the unoccupied levels are for down spins. When the TGF molecule is connected to electrodes, however, due to the chainTGF interactions energy level spectra is changed and it is not easy to exactly distinguish the contribution of TGF and electrode states.

In Figs. 3(b) and 3(c), we show the I-V characteristics of 4- and 5-TGFs for bare and singly hydrogenated cases. Hydrogenated flakes have lower conductance and leads to at least an order of magnitude smaller currents, since the hydrogenation of the flake removes some of the states providing open channels in the flake. While the maximum current in the calculated bias range is $\sim 5.00 \mu \mathrm{A}$ for bare TGF, after a-)

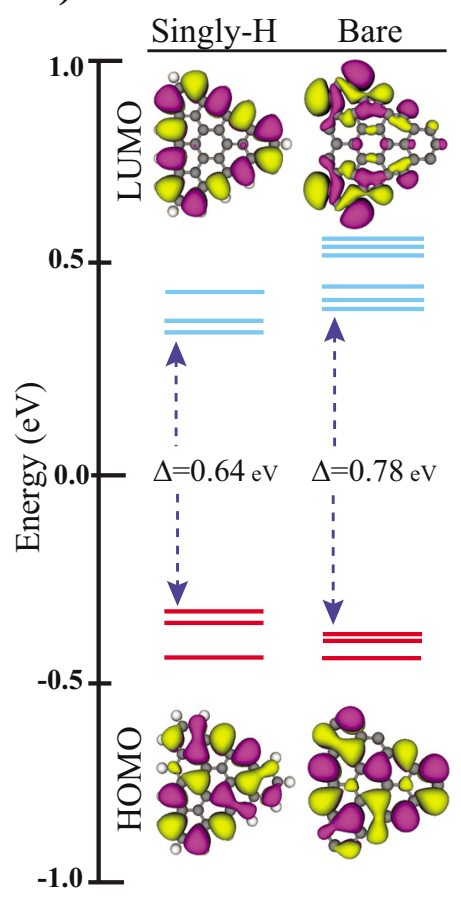

5-TGF
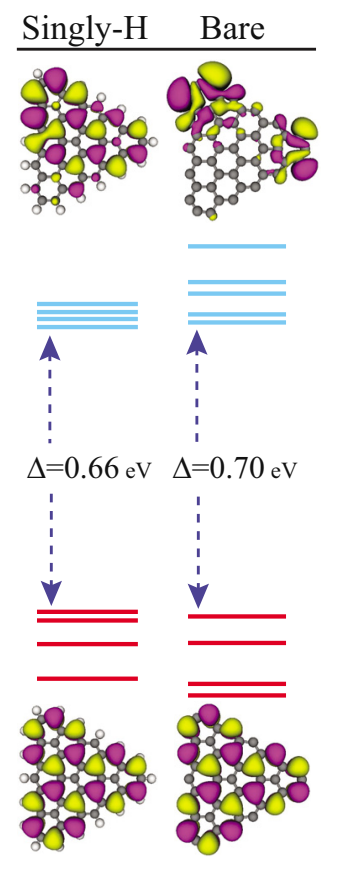

b-)
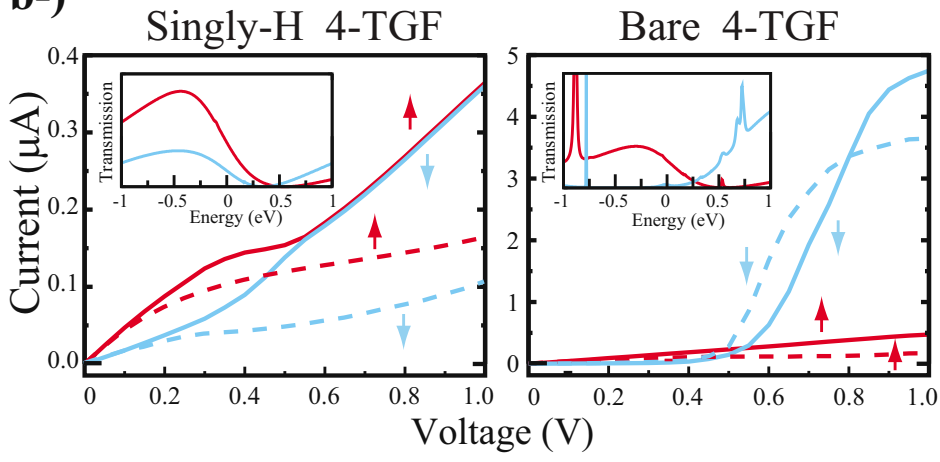

c-)

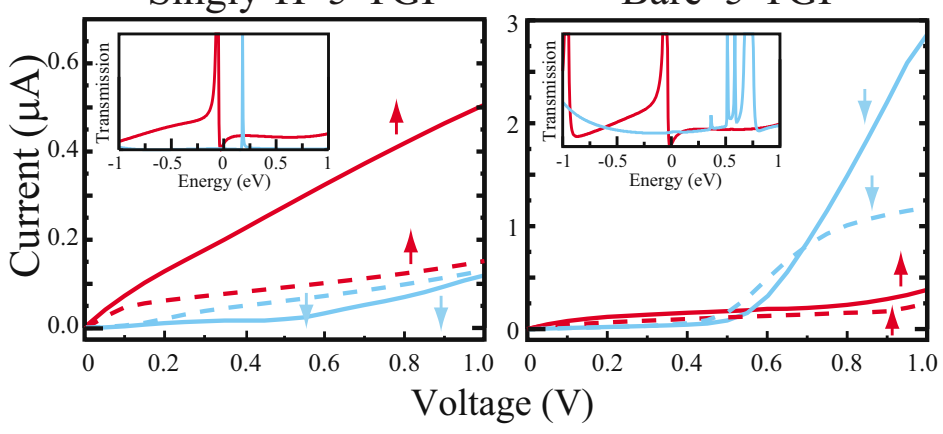

FIG. 3. (Color online) (a) Energy level spectra within $\pm 1 \mathrm{eV}$ range of Fermi level $\left(E_{F}\right)$, the HOMO-LUMO gap $(\Delta)$ and isosurface of HOMO and LUMO orbitals. [(b) and (c)] Calculated I-V curves for hydrogenated and bare TGFs. Results of 4-TGF and 5-TGF are presented, respectively. Spin-up ( $\uparrow$ ) and spin-down $(\downarrow)$ currents are shown by red (dark) and blue (light) lines, respectively. Solid and dashed lines denote forward and reverse bias calculations, respectively. Transmission curves of spin-up and spin-down under zero bias are also shown by insets. Transmission spectra of singly $\mathrm{H}$ and bare TGFs are plotted up to maximum value of 0.03 and 0.4 , respectively. Fermi levels are set to zero. 

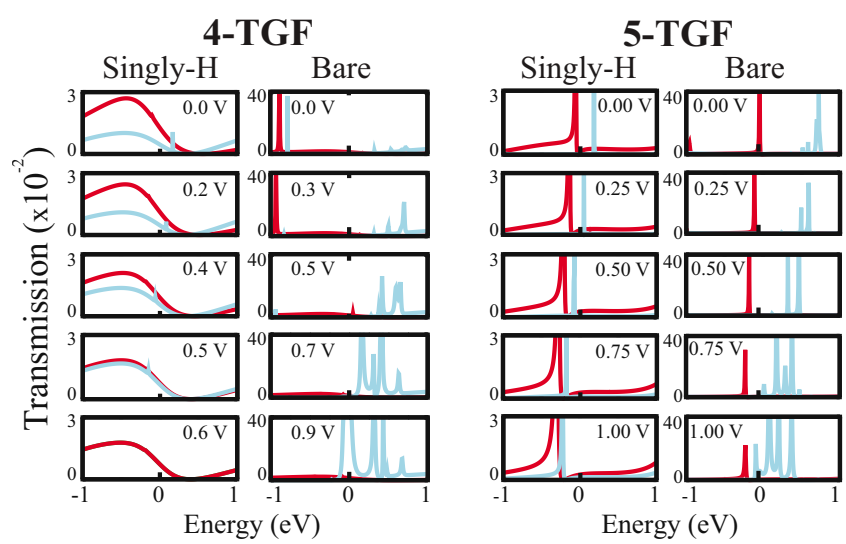

FIG. 4. (Color online) Bias dependent transmission spectra of singly hydrogen passivated (singly H) and bare TGFs for forward bias. Spin-up and spin-down transmissions are shown by red (dark) and blue (light) lines. $E_{F}$ is set to zero.

hydrogenation it is reduced to $\sim 0.35 \mu \mathrm{A}$. Since electrodedevice coupling broadens the energy levels, and may shift them due to charging, even though there is no molecular state at $\mathrm{E}_{F}$, the tails of HOMO and LUMO states can contribute to the transmission even at small voltages. Orbital character that changes upon the $\mathrm{H}$ termination of HOMO and LUMO states reveal the importance of the edge atoms in electron transport. In addition, there is a strong rectification of current for both singly $\mathrm{H}$ and bare edge cases. In this electrode-device configuration, forward current gets larger at a threshold bias of $\sim 0.6 \mathrm{~V}$. For small voltages, the characteristics of the current flowing through the TGFs can be understood by zero bias transmissions that are given by insets in Figs. 3(b) and 3(c). In fact, the self consistently calculated voltage dependent transmission spectra provides a better information regarding the I-V characteristics of the device.

Depending on the applied voltage, TGFs display diverse properties. In the case of forward bias application to the singly hydrogenated 4-TGF, the spin-up current is dominant up to $0.55 \mathrm{~V}$, but after this critical value, the spin-up and spindown states are merged due to the transition from ferromagnetic to antiferromagnetic state and thus spin polarizing property disappears. This behavior of I-V curves can be revealed from transmission spectrum. Even if the zero bias transmissions shown in insets in Figs. 3(b) and 3(c) can explain I-V curves for small bias values, at finite bias voltages the transmission spectrum of the flake changes through broadening and/or shifts in the transmission peaks, which should be calculated self-consistently under nonequilibrium conditions. In Fig. 4, we show the variations in transmission spectrum for selected cases under incremental forward bias voltages. For the hydrogenated 4-TGF, while transmission of the up spin channel decreases with increasing voltages, the transmission of down spins is increasing. Eventually both transmission curves are merging at the vicinity of $0.55 \mathrm{~V}$ and hence the spin polarization of the current is ceasing. Total magnetic moment of the flake together with electrodes has a bias dependence, gradually decreasing from $2 \mu_{B}$ to zero at the merging point of the up and down spin currents. In contrast, the down spin states of bare 4-TGF under forward bias get closer to the $E_{F}$ and become dominant carriers in the current. This explains how the spin polarization of the current is switched by applied bias. We can also establish a relation between I-V behavior and the corresponding transmission spectrum of bare 5-TGF. Since up and down spin transmission peaks do not show considerable variation within the energy window, the spin polarization of the currents is maintained up to $1 \mathrm{eV}$.

\section{CONCLUDING REMARKS}

In summary, we have investigated the electric, magnetic, and transport properties of triangle shaped graphene flakes. We have found that in addition to the their ferromagnetic ground state, TGFs show spin polarized and rectified current properties depending on edge saturation, flake size, bias voltage, and bias direction. Diverse and spin-dependent properties of graphene flakes depending on their shape, size, and edge saturation keep the promise of variety of application in future nanospintronics.

\section{ACKNOWLEDGMENTS}

This work was supported by TÜBITTAK under Grant No. 106T597, and through TR-Grid e-Infrastructure Project. Computing resources used in this work were partly provided by the National Center for High Performance Computing of Turkey (UYBHM) under Grant No. 2-024-2007.

${ }^{1}$ K. S. Novoselov, A. K. Geim, S. V. Morozov, D. Jiang, Y. Zhang, S. V. Dubonos, I. V. Grigorieva, and A. A. Firsov, Science 306, 666 (2004).

${ }^{2}$ K. S. Novoselov, A. K. Geim, S. V. Morozov, D. Jiang, M. I. Katsnelson, I. V. Grigorieva, S. V. Dubonos, and A. A. Firsov, Nature (London) 438, 197 (2005).

${ }^{3}$ Y. Zhang, Y.-W. Tan, H. L. Stormer, and Philip Kim, Nature (London) 438, 201 (2005).

${ }^{4}$ S. V. Morozov, K. S. Novoselov, M. I. Katsnelson, F. Schedin, D. C. Elias, J. A. Jaszczak, and A. K. Geim, Phys. Rev. Lett. 100, 016602 (2008).

${ }^{5}$ C. Lee, X. Wei, J. W. Kysar, and J. Hone, Science 321, 385 (2008).

${ }^{6}$ K. I. Bolotin, F. Ghahari, M. D. Shulman, H. L. Stormer, and P. Kim, Nature (London) 462, 196 (2009).

${ }^{7}$ F. Xia, T. Mueller, Y.-M. Lin, A. Valdes-Garcia, and P. Avouris, Nat. Nanotechnol. 4, 839 (2009)

${ }^{8}$ C. Chen, S. Rosenblatt, K. I. Bolotin, W. Kalb, P. Kim, I. Kymissis, H. L. Stormer, T. F. Heinz, and J. Hone, Nat. Nanotechnol. 4, 861 (2009)

${ }^{9}$ X. Wang, L. Zhi, and K. Mullen, Nano Lett. 8, 323 (2008).

${ }^{10}$ K. S. Kim, Y. Zhao, H. Jang, S. Y. Lee, J. M. Kim, K. S. Kim, J.-H. Ahn, P. Kim, J.-Y. Choi, and B. H. Hong, Nature (London) 457, 706 (2009).

${ }^{11}$ Q. Yan, B. Huang, J. Yu, F. Zheng, J. Zang, J. Wu, B.-L. Gu, F. Liu, and W. Duan, Nano Lett. 7, 1469 (2007).

${ }^{12}$ R. Murali, K. Brenner, Y. Yang, T. Beck, and J. D. Meindl, IEEE Electron Device Lett. 30, 611 (2009).

${ }^{13}$ H. Lee, Y. Son, N. Park, S. Han, and J. Yu, Phys. Rev. B 72, 174431 (2005).

${ }^{14}$ Y. Son, M. L. Cohen, and S. G. Louie, Nature (London) 444, 347 (2006).

${ }^{15}$ H. Şahin and R. T. Senger, Phys. Rev. B 78, 205423 (2008).

${ }^{16}$ M. Ezawa, Phys. Rev. B 76, 245415 (2007).

${ }^{17}$ O. Hod, J. E. Peralta, and G. E. Scuseria, Phys. Rev. B 76, 233401 (2007).

${ }^{18}$ O. V. Yazyev and L. Helm, Phys. Rev. B 75, 125408 (2007).

${ }^{19}$ J. J. Palacios, J. Fernandez-Rossier, and L. Brey, Phys. Rev. B 77, 195428 (2008).

${ }^{20}$ O. Hod, V. Barone, and G. E. Scuseria, Phys. Rev. B 77, 035411 (2008).

${ }^{21}$ J. Akola, H. P. Heiskanen, and M. Manninen, Phys. Rev. B 77, 193410 (2008).

${ }^{22}$ W. L. Wang, S. Meng, and E. Kaxiras, Nano Lett. 8, 241 (2008).

${ }^{23}$ M. R. Philpott, F. Cimpoesu, and Y. Kawazoe, Chem. Phys. 354, 1 (2008).

${ }^{24}$ A. V. Rozhkov and F. Nori, Phys. Rev. B 81, 155401 (2010).

${ }^{25}$ H. Şahin, C. Ataca, and S. Ciraci, Appl. Phys. Lett. 95, 222510 (2009).

${ }^{26}$ D. C. Elias, R. R. Nair, T. M. G. Mohiuddin, S. V. Morozov, P. Blake, M. P. Halsall, A. C. Ferrari, D. W. Boukhvalov, M. I. Katsnelson, A. K. Geim, 
and K. S. Novoselov, Science 323, 610 (2009).

${ }^{27}$ J. O. Sofo, A. S. Chaudhari, and G. D. Barber, Phys. Rev. B 75, 153401 (2007).

${ }^{28}$ D. W. Boukhvalov, M. I. Katsnelson, and A. I. Lichtenstein, Phys. Rev. B 77, 035427 (2008).

${ }^{29}$ M. Z. S. Flores, P. A. S. Autreto, S. B. Legoas, and D. S. Galvao, Nanotechnology 20, 465704 (2009).

${ }^{30}$ H. Şahin, C. Ataca, and S. Ciraci, Phys. Rev. B 81, 205417 (2010).

${ }^{31}$ E. Bekyarova, M. E. Itkis, P. Ramesh, C. Berger, M. Sprinkle, W. A. de Heer, and R. C. Haddon, J. Am. Chem. Soc. 131, 1336 (2009).

${ }^{32}$ D. W. Boukhvalov and M. I. Katsnelson, Phys. Rev. B 78, 085413 (2008).

${ }^{33}$ Q.-X. Pei, Y.-W. Zhang, and V. B. Shenoy, Nanotechnology 21, 115709 (2010).

${ }^{34}$ J. H. Warner, M. H. Rummeli, L. Ge, T. Gemming, B. Montanari, N. M. Harrison, B. Buchner, and G. A. D. Briggs, Nat. Nanotechnol. 4, 500 (2009).

${ }^{35}$ J. C. Meyer, C. O. Girit, M. F. Crommie, and A. Zettl, Nature (London) 454, 319 (2008).

${ }^{36}$ X. Jia, M. Hofmann, V. Meunier, B. G. Sumpter, J. Campos-Delgado, J.
M. Romo-Herrera, H. Son, Y.-P. Hsieh, A. Reina, J. Kong, M. Terrones, and M. S. Dresselhaus, Science 323, 1701 (2009).

${ }^{37}$ L. Tapasztó, G. Dobrik, P. Lambin, and L. P. Biró, Nat. Nanotechnol. 3, 397 (2008)

${ }^{38}$ M. Y. Han, B. Ozyilmaz, Y. Zhang, and P. Kim, Phys. Rev. Lett. 98, 206805 (2007).

${ }^{39}$ C. K. Gan and D. J. Srolovitz, Phys. Rev. B 81, 125445 (2010).

${ }^{40}$ O. U. Aktürk and M. Tomak, Appl. Phys. Lett. 96, 081914 (2010).

${ }^{41}$ W. Zhang, L. Sun, Z. Xu, A. V. Krasheninnikov, P. Huai, Z. Zhu, and F. Banhart, Phys. Rev. B 81, 125425 (2010).

${ }^{42}$ P. Koskinen, S. Malola, and H. Häkkinen, Phys. Rev. B 80, 073401 (2009).

${ }^{43}$ A. Kuc, T. Heine, and G. Seifert, Phys. Rev. B 81, 085430 (2010).

${ }^{44}$ Distributed by QuantumWise company, Copenhagen, Denmark. http:// www.quantumwise.com

${ }^{45}$ E. H. Lieb, Phys. Rev. Lett. 62, 1201 (1989).

${ }^{46}$ S. Tongay, R. T. Senger, S. Dag, and S. Ciraci, Phys. Rev. Lett. 93, 136404 (2004) 